\title{
A propensity-matched analysis of stereotactic body radiotherapy and sublobar resection for stage I non-small cell lung cancer in patients at high risk for lobectomy: the results in a Chinese population
}

\author{
Xiao-Shuai Yuan ${ }^{1 \#}$, Wu-Cheng Chen ${ }^{2 \#}$, Qing-Ren Lin ${ }^{3 \#}$, Yuan-Jun Liu ${ }^{3}$, Yao-Yao Zhu ${ }^{1}$, Xiao-Jiang Sun ${ }^{2}$, \\ Qiong-Ya Wu ${ }^{1}$, Jin-Shi Liu ${ }^{4}$, Ya-Ping $\mathrm{Xu}^{1,2,3}$ \\ ${ }^{1}$ Department of Radiation Oncology, Shanghai Pulmonary Hospital Tongji University, Shanghai, China; ${ }^{2}$ First Clinical Medical School, Wenzhou \\ Medical University, Wenzhou, China; ${ }^{3}$ Department of Radiation Oncology, Cancer Hospital, University of Chinese Academy of Sciences, Hangzhou, \\ China; ${ }^{4}$ Department of Thoracic Oncology, Cancer Hospital, University of Chinese Academy of Sciences, Hangzhou, China \\ Contributions: (I) Conception and design: XS Yuan, WC Chen, QR Lin, QY Wu, JS Liu, YP Xu; (II) Administrative support: YP Xu; (III) Provision \\ of study materials or patients: QR Lin, XJ Sun, JS Liu; (IV) Collection and assembly of data: YJ Liu, YY Zhu, XJ Sun; (V) Data analysis and \\ interpretation: XS Yuan, WC Chen, QR Lin, XJ Sun, JS Liu; (VI) Manuscript writing: All authors; (VII) Final approval of manuscript: All authors. \\ \#These authors contributed equally to this work. \\ Correspondence to: Prof. Ya-Ping Xu. Department of Radiation Oncology, Shanghai Pulmonary Hospital Tongji University, No. 507 Zhengmin Road, \\ Shanghai, China. Email: dr_xuyaping@163.com; Prof. Jin-Shi Liu. Department of Thoracic Oncology, Cancer Hospital, University of Chinese \\ Academy of Sciences, No. 1 East Road Banshan, Hangzhou, China. Email: yefeng9725boy@163.com; Prof. Qiong-Ya Wu. Department of Radiation \\ Oncology, Shanghai Pulmonary Hospital Tongji University, No. 507 Zhengmin Road, Shanghai, China. Email: wqyluj@sohu.com.
}

Background: To investigate the comparative effectiveness of stereotactic body radiotherapy (SBRT) and sublobar resection (SLR) in patients with stage I non-small cell lung cancer (NSCLC) considered to be highrisk lobectomy patients.

Methods: From January 2012 to December 2015, patients who underwent SBRT or SLR for clinical stage I NSCLC were examined retrospectively. Propensity score matching (PSM) was performed to reduce selection bias in SBRT and SLR patients.

Results: Data from 86 SBRT and 79 SLR patients was collected. Median follow-up periods of the SBRT and SLR groups were 32 and 37 months, respectively. Patients treated with SBRT exhibited significantly higher age, higher likelihood of being male, larger tumor diameter, lower forced expiratory volume in 1 second (FEV1), and poorer performance status compared with SLR patients. There were no significant differences between SBRT and SLR patients for 3-year overall survival (OS) (80.3\% and 82.3\%, P=0.405), cause-specific survival (CSS) (81.3\% and 83.4\%, $\mathrm{P}=0.383)$, and local control (LC) $(89.7 \%$ and $86.0 \%$, $\mathrm{P}=0.501)$. Forty-nine patients were identified from each group after performing PSM. After patients were matched for age, gender, performance status, tumor characteristics and pulmonary function, no significant differences were observed in 3-year OS (85.4\% and 73.3\%, $\mathrm{P}=0.649)$, CSS (87.2\% and $74.9 \%, \mathrm{P}=0.637)$ and LC (95.6\% and $82.1 \%, \mathrm{P}=0.055)$. Prevalence of significant adverse events (grade 3 or worse) was $0 \%$ and $10.2 \%$ in the matched SBRT and SLR groups ( $\mathrm{P}=0.056)$, respectively.

Conclusions: Disease control and survival in the SBRT patients was equivalent to that seen in SLR patients with stage I NSCLC considered high-risk lobectomy candidates. SBRT could therefore be an alternative option to SLR in treating patients with a high operative risk.

Keywords: Non-small cell lung cancer (NSCLC); stereotactic body radiotherapy (SBRT); sublobar resection (SLR); propensity score

Submitted Jan 27, 2021. Accepted for publication Mar 18, 2021.

doi: $10.21037 /$ jtd-21-339

View this article at: http://dx.doi.org/10.21037/jtd-21-339 


\section{Introduction}

Lung cancer is a leading cause of cancer-related death worldwide (1). The incidence of early-stage non-small cell lung cancer (NSCLC) is increasing due to increased tobacco use, an aging population profile, and advances in computed tomography(CT)-based screening (2-4). Lobectomy with systematic lymph node evaluation is a recommended strategy for treating standard-surgical-risk patients with early-stage NSCLC, showing optimal disease control and survival compared with stereotactic body radiotherapy (SBRT) and sublobar resection (SLR) (5-8). Unfortunately, in stage I NSCLC patients, approximately $40 \%$ of elderly patients-as well as those with impaired cardiopulmonary function, medical comorbidities, or not wanting surgical intervention-do not undergo lobectomy (9). Alternate treatment options are therefore essential for these high-risk surgical candidates, and effective treatment methods need to be explored to achieve better survival outcomes.

During SLR, less lung parenchyma tissue is removed compared with lobectomy, leading to significantly better functional preservation (10). In recent years, the introduction of video-assisted thoracoscopic surgery (VATS) for SLR has reduced many of the perioperative morbidities associated with thoracotomy. SLR is a primary therapy for treating high-risk surgical patients. Although controversial, some retrospective studies have even suggested that the outcomes of SLR - especially anatomical segmentectomymay be similar to that of lobectomy for patients with peripheral small-sized tumors (lesion $\leq 2 \mathrm{~cm})(11-14)$. SBRT-also known as stereotactic ablative radiation (SABR) - is another important alternative to surgery, emerging as an effective and well-tolerated intervention for stage I NSCLC patients in medically inoperable patients (15-17). Studies have demonstrated that SBRT has better local control and longer overall survival (OS) in inoperable stage I NSCLC patients compared with conventional radiotherapy $(18,19)$. The application of SBRT has ranged from medically inoperable to potentially operable patients $(20,21)$, and the survival rate of SBRT is comparable to surgery. Therefore, SBRT and SLR are considered as the main alternative therapies for treating stage I NSCLC patients with high surgical risk.

A phase III randomized study [the American College of Surgeons Oncology Group (ACOSOG) Z4099/the Radiation Therapy Oncology Group (RTOG) 1021] compared SBRT with SLR in stage I NSCLC patients with high surgical risk (22) but was closed due to poor recruitment. Currently, the outcomes of prospective randomized clinical trials are limited, and the optimal therapeutic regime for stage I NSCLC patients with high surgical risk is still unclear.

The aim of this retrospective study was to compare outcomes of SBRT and SLR using propensity score matching (PSM) analysis among stage I NSCLC patients with high surgical risk. We investigated the current evidence in the application of SBRT and SLR in stage I NSCLC to help guide clinical decision-making in the absence of a randomized controlled trial. We present the following article in accordance with the STROBE reporting checklist (available at http://dx.doi.org/10.21037/jtd-21-339).

\section{Methods}

\section{Patient evaluation}

A retrospective analysis was performed using data collected from Zhejiang Cancer Hospital, Hangzhou, PR China. All consecutive patients with histologically-confirmed stage I NSCLC who underwent SBRT or SLR from January 2012 to December 2015 were examined. The patients enrolled in the study satisfied the following eligibility criteria: (I) diagnosis of T1-2a (tumor size less than $5 \mathrm{~cm}$ ) N0M0 NSCLC according to the American Joint Committee on Cancer (AJCC) $7^{\text {th }}$ Edition Staging Criteria; (II) ineligibility for anatomic lobectomy; (III) Eastern Cooperative Oncology Group (ECOG) performance status (PS) $\leq 2$. The exclusion criteria were: (I) pure ground glass opacities on imaging; (II) pathology confirmation after SLR of more advanced stage than T1-2a N0M0; (III) previous neoadjuvant chemotherapy or thoracic radiation therapy; (IV) no pre-treatment pulmonary function test results available. The study is conformed to the provisions of the Declaration of Helsinki (as revised in 2013), and all participants were informed consent before taking part in the research. The Ethics Review Committee of Zhejiang Cancer Hospital approved this study.

Clinical stage and treatment plan were determined by a multidisciplinary oncology team, consisting of a radiation oncologist, thoracic surgeon, medical oncologist, pathologist and diagnostic radiologist. The clinical staging of the tumor size $(\mathrm{T})$ and lymph node involvement $(\mathrm{N})$ was determined based on routine CT findings. $18 \mathrm{~F}$-fluorodeoxyglucose positron emission tomography/computed tomography (FDG-PET/CT) was performed where patients voluntarily received pre-treatment PET/CT staging or where there 
was high suspicion of lymph node involvement or distant metastases. Age-independent Charlson Comorbidity Index (CCI) was used to record comorbidity scores (23). Therapeutic toxicity was graded according to the Common Terminology Criteria of Adverse Events (version 4.0). Recurrence types were classified according to RTOG 1021/ ACOSOG Z4099. Local recurrence was defined as tumor recurrence at the primary site, staple line or involved lobe, while regional recurrence was defined as ipsilateral nodal recurrence in the hilum or mediastinum, or recurrence in the ipsilateral lung. Distant recurrence referred to recurrence beyond the local or regional recurrence criteria. Recurrence was clinically determined by tissue biopsy or by a radiation oncologist based on PET/CT or CT alone. OS was defined as the period from the beginning of treatment to death or to final follow-up. Cause-specific survival (CSS) was the interval from the beginning of treatment to cancerrelated death, treatment-related death, or final followup. Recurrence-free survival (RFS) was defined as the interval from the beginning of treatment to death, or any recurrence, or final follow-up.

\section{Treatment}

SLR was mainly performed through VATS; however, where VATS was not deemed appropriate based on pre- or intra-operative judgement, thoracotomy was performed. The decision to perform segmentectomy or wedge resection - with or without hilar and mediastinal lymph node dissection-was made by the surgeon based on tumor location, size, generous margins, surgical risk, and intraoperative findings. Distance from the dissection margin to tumor edge was longer than the maximum tumor diameter or $20 \mathrm{~mm}$. All removed lymph nodes were confirmed as negative for metastatic disease by frozen section analysis. The negative resection margins were histologically confirmed before completion of surgery.

SBRT patients were immobilized with a vacuum pillow. Four-dimensional CT (4D-CT) accompanied by real-time position management was used. Respiratory gating was used if tumor motion was greater than $15 \mathrm{~mm}$ on 4D-CT. Gross tumor volume (GTV) was delineated in ten phases (from $0 \%$ to $90 \%$ breathing phases) which were combined to ascertain internal target volume (ITV). The ITV was uniformly expanded by $5 \mathrm{~mm}$ to generate the planning target volume (PTV). Dose calculation was performed on the average intensity projection (AIP), which were optimized by using 9-15 coplanar or non-coplanar 6-MV photon beams and also can be used as the reference for image guidance.

\section{Statistical analysis}

A PSM analysis was performed to match baseline characteristics of SBRT and SLR patients. Patients were matched based on the following covariates: age, gender, performance status, tumor diameter, cancer stage, and forced expiratory volume in 1 second (FEV1). Differences in patient baseline characteristics between treatment groups or subgroups were assessed using Mann-Whitney $U$ test and chi-square test for continuous and categorical data, respectively. Survival probability was evaluated using the Kaplan-Meier estimator, while survival differences between groups and subgroups were studied using a log-rank test.

Statistical analyses were performed using IBM Statistic Package for Social Science software (version 22.0; SPSS, Inc., Chicago, IL, USA). Statistical significance was defined as $\mathrm{P}<0.05$.

\section{Results}

\section{Patient characteristics}

From January 2012 to December 2015, 86 patients who underwent SBRT and 79 patients who underwent SLR (including 35 wedge resection and 44 segmentectomy) were recruited into the study. Median age at diagnosis was 75 years in the SBRT group and 65 years in the SLR group. A summary of characteristics of patients is shown in Table 1. Compared with those who received SLR, patients treated with SBRT were significantly older with poorer PS, lower FEV1 and larger tumor diameter. A significantly higher proportion of male patients were treated with SBRT than SLR $(\mathrm{P}=0.019)$. There was no significant difference in disease stage between SBRT and SLR groups $(\mathrm{P}=0.295)$; however, there was a statistically significant difference in the histological subtypes between groups $(\mathrm{P}=0.030)$. In the SBRT group, adenocarcinomas, squamous cell carcinomas and other tumor types accounted for $46.9 \%$, $30.6 \%$ and $22.4 \%$, respectively, while in the SLR group, adenocarcinomas, squamous cell carcinomas and other tumors accounted for $69.4 \%, 24.5$ and $6.1 \%$, respectively.

A propensity score analysis was performed in the SBRT and SLR groups. 49 patients were identified from each group; matched SBRT and SLR patients showed no significant differences in age, gender, PS, tumor size, FEV1, or cancer stage (Table 1). Patients in these groups 
Table 1 Characteristics of all patients and propensity score matched patients

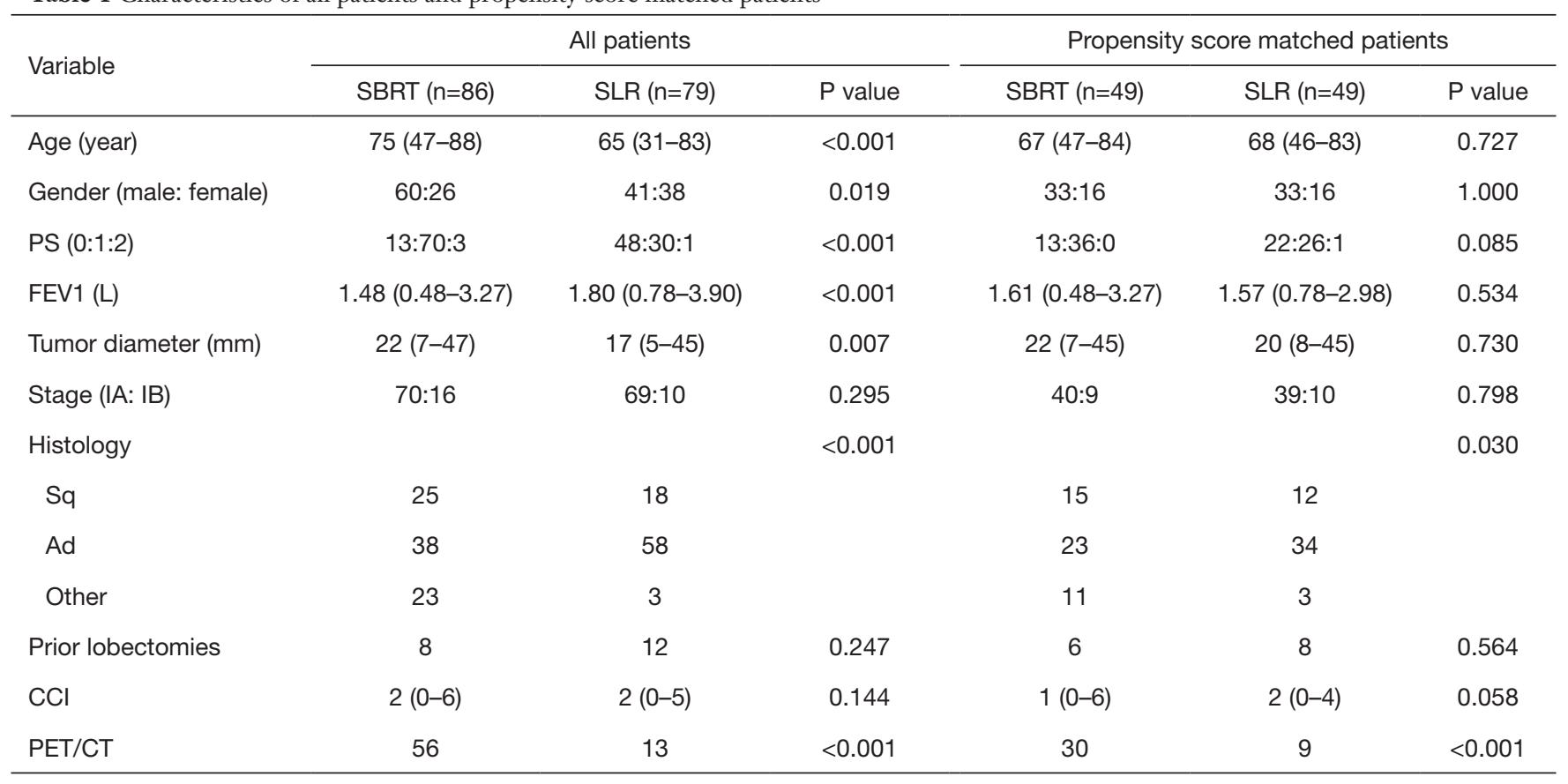

Values are shown in median (range) for continuous data. SBRT, stereotactic body radiotherapy; SLR, sublobar resection; PS, performance status; FEV1, forced expiratory volume in 1 second; Sq, squamous cell carcinomas; Ad, adenocarcinomas; CCI, Charlson Comorbidity Index; PET/CT, positron emission tomography/computed tomography.

who had undergone previous lobectomy also showed no significant differences in these characteristics at baseline ( 6 and 8 patients, respectively, $\mathrm{P}=0.564)$. FDGPET/CT was performed in 30 (61.2\%) SBRT patients, compared with $9(18.4 \%)$ SLR patients $(\mathrm{P}<0.001)$. Of the SLR patients, 26 underwent segmentectomy, with the remaining 23 undergoing wedge resection. Lymphnode systematic dissection or sampling was performed in $22(84.6 \%)$ segmentectomy patients and 4 (17.4\%) wedge resection patients; the remaining 4 and 19 patients in each respective group did not undergo lymph node dissection, as there was no preoperative or intraoperative suspicion for lymph node involvement. VATS was performed in 41 (83.7\%) SLR cases, of which 2 (4.1\%) required conversion to thoracotomy during surgery. SBRT patients were predominantly treated with a 50-Gy prescribed dose in five fractions, with a biological effective dose (BED) of $100 \mathrm{~Gy}$ $(\alpha / \beta=10)$.

\section{Survival and recurrence differences}

Prior to performing PSM, the median follow-up periods of the SBRT and SLR groups were 32 and 37 months, respectively. The median OS in each group was not reached at the time of final follow-up. During the follow-up period, 21 SBRT and 16 SLR patients died: 34 patients died of lung cancer and 3 died of other causes. These included one case of treatment-related death due to cardiac tamponade following SLR, one death following fracture 6.87 months after SLR (segmentectomy), and one death from pneumonia 2 months after SBRT. There were no significant differences between groups for OS $(80.3 \%$ and $82.3 \%$ for 3 -year OS, $\mathrm{P}=0.405)$ (Figure $1 A)$ and CSS $(81.3 \%$ and $83.4 \%$ for 3 -year $\mathrm{CCS}, \mathrm{P}=0.383$ ) (Figure $1 B$ ).

At the time of the final follow-up, 28 SBRT and 25 SLR patients had experienced disease recurrence, and there was no significant difference in RFS between groups $(62.4 \%$, and $69.9 \%$ for 3 -year RFS, $\mathrm{P}=0.383)$. In the SBRT and SLR groups, respectively, there were 8 and 11 cases of local recurrence, 10 and 10 cases of regional recurrence, and 22 and 21 cases of distant recurrence. The rates of local (Figure 2), regional and distance control were not significantly different between groups $(89.7 \%$ and $86.0 \%$ for 3 -year local control, $\mathrm{P}=0.501 ; 87.1 \%$ and $87.9 \%$ for 3 -year regional control, $\mathrm{P}=0.884$ and $71.6 \%$ and $74.4 \%$ for 3 -year distance control, $\mathrm{P}=0.662$, respectively). 
A

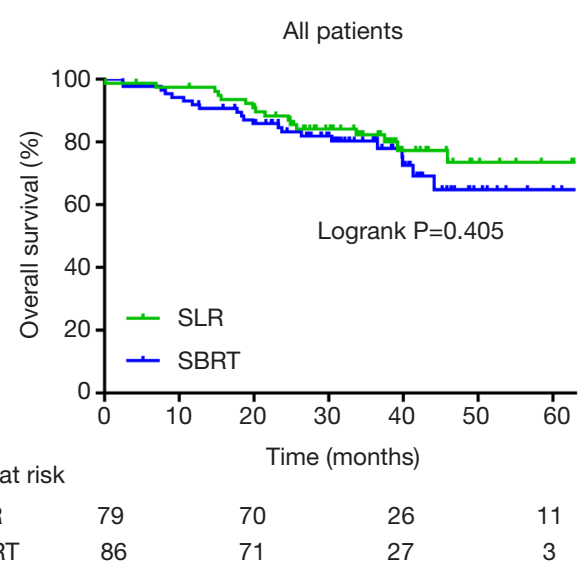

B

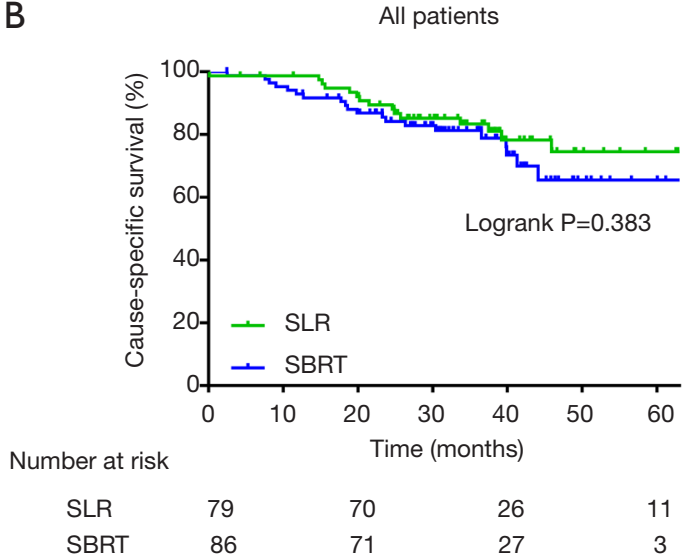

Figure 1 The survival in all patients. (A) Overall survival in all patients; (B) cause-specific survival in all patients.

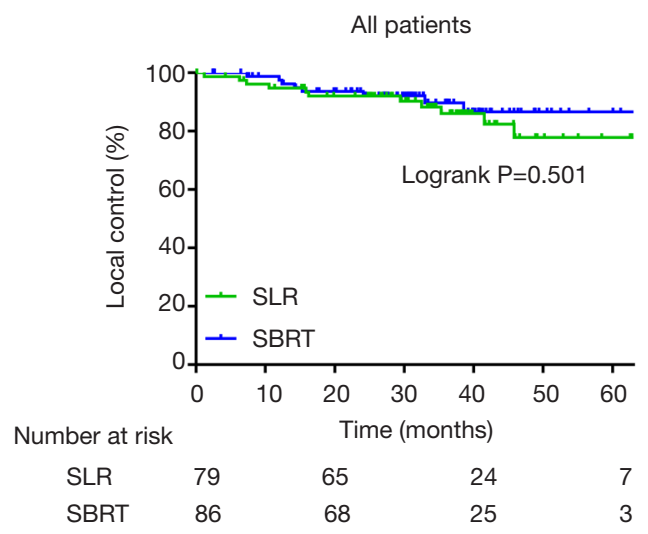

Figure 2 Local control in all patients.

After performing PSM, 10 SBRT patients and 13 SLR

patients died before final follow-up; among them, 9 SBRT
A

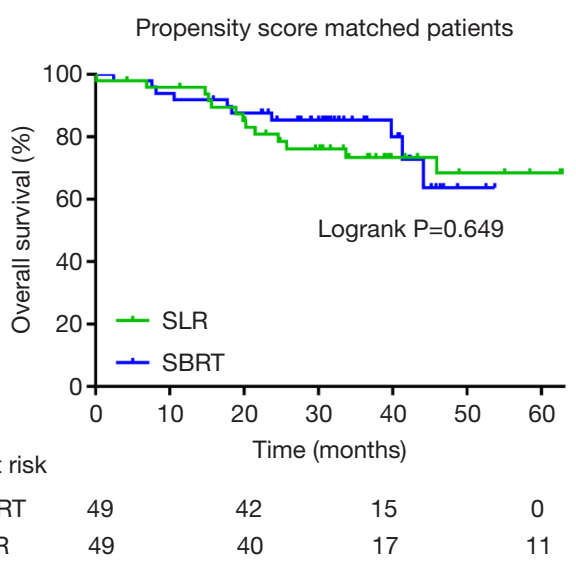

B

Propensity score matched patients

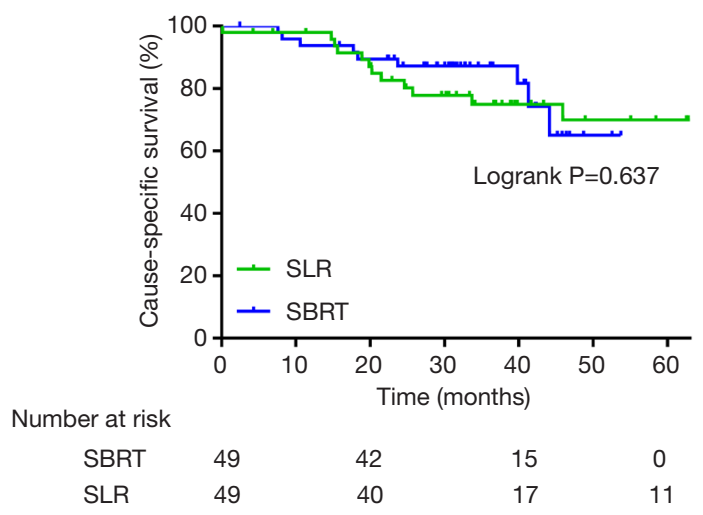

Figure 3 The survival in propensity score matched patients. (A) Overall survival in PSM patients; (B) cause-specific survival in PSM patients. PSM, propensity score matching.

and 11 SLR patients died of lung cancer recurrence, while 3 died of other causes. No statistically significant difference between groups was identified for OS $(85.4 \%$ and $73.3 \%$ for 3-year OS, $\mathrm{P}=0.649)$ (Figure $3 A$ ) and CSS $(87.2 \%$ and $74.9 \%$ for 3 -year CCS, $\mathrm{P}=0.637$ ) (Figure $3 B$ ).

There were 14 SBRT and 19 SLR patients in the matched groups who experienced disease recurrence, and the difference between the two matched groups in RFS was not significant $(66.7 \%$, and $63.2 \%$ for 3 -year RFS, $\mathrm{P}=0.689)$. In the matched SBRT and SLR groups, there were 2 and 9 cases of local recurrence, 4 and 8 cases of regional recurrence, and 11 and 16 cases of distant recurrence, respectively. The rates of local (Figure 4), regional and distance control were not significantly different $(95.6 \%$ and $82.1 \%$ for 3 -year local control, $\mathrm{P}=0.055 ; 91.4 \%$ and $85.2 \%$ for 3 -year regional control, $\mathrm{P}=0.432$; and $73.7 \%$ and $68.3 \%$ for 3 -year distance control, $\mathrm{P}=0.548$, respectively). 
In a subgroup analysis of matched patients, the number of patients was limited in wedge resection and segmentectomy subgroups. No significant difference was identified in OS, CSS, RFS, regional control, and distance control between SBRT and segmentectomy or between SBRT and wedge resection $(\mathrm{P}>0.05)$. Local control of SBRT patients was better than those who underwent wedge resection $(95.6 \%$ versus $67.4 \%$ for 3 -year local control, $\mathrm{P}=0.005)$. Compared with those who underwent wedge resection, patients treated with segmentectomy had a tendency of improved 3-year local control $(95.7 \%$ versus $67.4 \% ; \mathrm{P}=0.075)$ and $\mathrm{RFS}$ (77.2\% versus $49.3 \%, \mathrm{P}=0.076$ ); however, patients who had wedge resection had a higher CCI compared with those received segmentectomy $(\mathrm{P}=0.013)$.

\section{Treatment toxicity}

Post-treatment adverse events in the matched SBRT and SLR patients are shown in Tables 2,3. In the SBRT cohort,

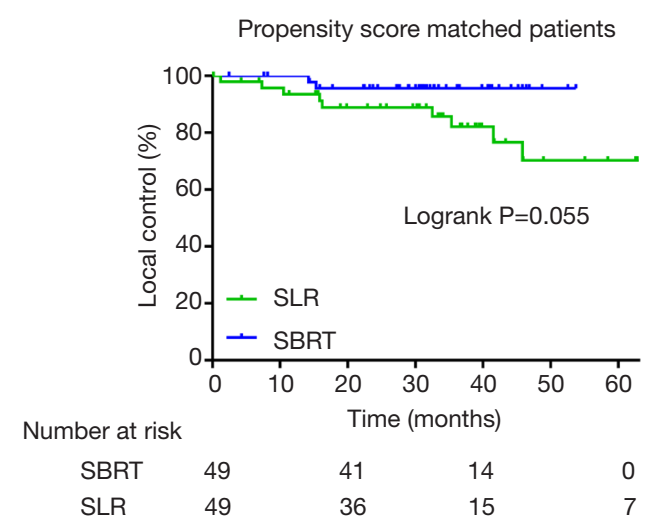

Figure 4 Local control in propensity score matched patients. seven $(14.3 \%)$ patients experienced grade 2 respiratory adverse events; specifically, there were six cases $(12.2 \%)$ of radiation pneumonitis and one case $(2.0 \%)$ of cough, which were all respiratory complications. No adverse events of grade 3 or worse were observed in the SBRT group. In the SLR cohort, 13 patients (26.5\%) had grade 2 or worse adverse events. Eight patients (16.3\%) experienced grade 2 adverse events-including five cases $(10.2 \%)$ of dyspnea and three cases $(6.1 \%)$ of chest wall pain-while grade 3 , 4 and 5 adverse events occurred to 5 patients (10.2\%)including two cases $(4.1 \%)$ of atrial fibrillation, one case (2.0\%) of pulmonary thromboembolism, one case of $(2.0 \%)$ of hypoxia and one case $(2.0 \%)$ of pericardial tamponade. Grade 3 or worse adverse events after SLR were frequently cardiovascular complications. The rate of grade 3 or worse adverse events was 0 and $10.2 \%$ in matched SBRT and SLR groups, respectively $(\mathrm{P}=0.056)$.

\section{Discussion}

SBRT is a preferred strategy for treating medically inoperable patients with stage I NSCLC. For patients with a high operative risk, SLR is commonly used as a potential alternative to open lobectomy, while SBRT is also encouraged to be discussed within the multidisciplinary cancer care team (24). To date, no phase III randomized controlled trials comparing SBRT to SLR in high-risk surgical patients have been completed. Patients treated with SBRT are typically medically inoperable, or, in some cases, potentially operable. A retrospective direct comparison of outcomes between SBRT and SLR is problematic due to the heterogeneity of this population (25). Furthermore, defining operative risk is complicated and subjective, especially for patients with a multiple comorbidities,

Table 2 Posttreatment adverse events in the matched SBRT patients

\begin{tabular}{lrr}
\hline AEs & CTCAE grade & SBRT $(\mathrm{n}=49)(\%)$ \\
\hline Fatigue & 1 & $1(2.0)$ \\
Chest wall pain & 1 & $1(2.0)$ \\
Dyspnea & 1 & $4(8.2)$ \\
Cough & 1 & $11(22.4)$ \\
& 2 & $1(2.0)$ \\
Radiation pneumonitis & 1 & $13(26.5)$ \\
\end{tabular}

SBRT, stereotactic body radiotherapy. 
Table 3 Posttreatment adverse events in the matched SLR patients

\begin{tabular}{lcc}
\hline AEs & CTCAE grade & SLR (n=49) $(\%)$ \\
\hline Fatigue & 1 & $8(16.3)$ \\
Anorexia & 1 & $1(2.0)$ \\
Chest wall pain & 1 & $3(6.1)$ \\
& $24.5)$ & $11(22.4)$ \\
Dyspnea & 1 & $5(10.2)$ \\
Cough & 2 & $11(22.4)$ \\
Atrial fibrillation & 1 & $2(4.1)$ \\
Hypoxia & 3 & $1(2.0)$ \\
Pulmonary thromboembolism & 4 & $1(2.0)$ \\
Pericardial tamponade & 4 & $1(2.0)$ \\
\hline
\end{tabular}

SLR, sublobar resection.

impaired cardiopulmonary function, and advanced age. The assessment of operative risk is frequently discussed within a multidisciplinary team and based on clinical judgement, therefore it may not always meet the strict high-risk and inoperable criteria reported in prospective clinical trials (ACOSOG Z4032 and RTOG 0236) (16,26). With the development of surgical technology and the appearance of new surgical methods, the risk of operation is constantly reduced. The comparison between the new surgical technology and SBRT requires further study. The use of PSM in a retrospective study can produce more comparable groups by comparing the outcomes of SBRT and SLR in high-risk operable patients with stage I NSCLC.

Some retrospective studies have also attempted to use PSM to balance selection bias of a population in the comparison of survival in SBRT and SLR. These studies have been conducted based on institution case series or administrative data from large databases including the Surveillance, Epidemiology, and End Results (SEER) database and the National Cancer Database (NCDB). Port et al. (27) reviewed 164 patients who underwent wedge resection or SBRT with clinical stage IA NSCLC, and 99 patients were matched by age, sex, and histology; no significant difference was identified in OS between the wedge resection and SBRT groups (3-year OS $87 \%$ vs. $75 \%$, respectively; $\mathrm{P}=0.357$ ). Matsuo et al. (28) performed a survival comparison between SBRT and SLR in patients who had stage I NSCLC and were considered to be high-risk lobectomy candidates. 115 SBRT and 65 SLR patients were enrolled and the results showed that, before performing PSM, the 5-year OS was short in the SBRT than the SLR group (40.3\% and $60.5 \%$, respectively; $\mathrm{P}=0.008)$ and cause-specific death was not significantly different. Meanwhile, after performing PSM-whereby 53 patients were matched from each treatment group in terms of age, gender, performance status, tumor diameter, FEV1, and CCI-there was no significant difference between groups for 5 -year OS $(40.4 \%$ and $55.6 \%$, respectively, $\mathrm{P}=0.124)$ and cause-specific death $(35.3 \%$ and $30.3 \%$, respectively; $\mathrm{P}=0.427$ ). Shirvani et al. (9) used the SEER database to compare survival outcomes between SLR and SBRT patients age over 66 years with stage I NSCLC and showed that lung cancer-specific survival (LCSS) was not significantly different between the matched SLR and SBRT groups (OS HR 0.82, 95\% CI, 0.53-1.27, P=0.38; LCSS HR 2.14, 95\% CI, 0.87-5.26, $\mathrm{P}=0.10$ ). Ezer et al. (29) also performed a retrospective analysis using the SEER database which included patients aged above 65 years old with stage I-II NSCLC N0 treated with SBRT or SLR; after PSM, this study showed no differences between groups in OS (HR; $1.20,95 \%$ CI: $0.98-1.49$ ) and cancer-specific survival (HR: 1.48, 95\% CI: 0.97-2.42). Our results were consistent with the conclusions from these previous reports, as SBRT achieved comparable survival outcomes compared with SLR after PSM. Thus, our data supported the fact that SBRT could be used as an alternative treatment to SLR.

Conversely, there were two additional population-based analyses that were inconsistent with our findings. Using 
the SEER database, Paul et al. (30) compared the survival outcomes of thoracoscopic SLR and SBRT and found that in patients aged above 66 years with stage I NSCLC (tumor size $\leq 2 \mathrm{~cm}$ ) there was no significant difference between matched groups for CSS (HR 1.32, 95\% CI, 0.77 to 2.26; $\mathrm{P}=0.32$ ) but that the SLR group had a significantly better OS compared with SBRT (HR 1.80, 95\% CI, 1.33 to 2.43; $\mathrm{P}<0.001$ ). Furthermore, Yerokun et al. (31) used the National Cancer Database to compare outcomes between wedge resection and SBRT patients with stage clinicalT1N0M0 (tumors $\leq 2 \mathrm{~cm}$ ) NSCLC, and found that SBRT was associated with significantly reduced 5-year survival, compared with wedge resection in both unmatched analysis $(30.9 \%$ vs. $55.2 \%, \mathrm{P}<0.001)$ and after PSM analysis (31.0\% vs. $49.9 \%, \mathrm{P}<0.001)$. Therefore, using SBRT as an alternative treatment option to SLR for treating high-risk operable patients is still controversial, thus, a prospective study is required to determine survival rates in SBRT and SLR.

Both SBRT and SLR have inherent disadvantages over lobectomy in terms of disease control. SBRT delivers a very precise and high dose of radiation to a lung tumor in a small number of fractions and achieves more than $90 \%$ of local control rate with prescribed dose of BED $\geq 100$ Gy $(15,17)$; however, the tumor is still not removed. Low prescription dose of BED and insufficient dose coverage due to the uncertainty of internal tumor movement and/or patient set-up results in worse local control (32-34). SLR increases inadequate or positive surgical margins and significantly increases the possibility of local recurrence compared with lobectomy (5). Furthermore, both SBRT and SLR do not remove unsuspected intralobar tumor spread. It is still unclear whether SBRT or SLR performs better in disease control. Matsuo et al. (28) reported a higher local recurrence after SBRT compared with SLR (28.3\% and $14.1 \%$ at 5 years, $\mathrm{P}=0.059)$. Port et al. (27) showed that overall recurrence (local and distant) was significantly higher after SBRT (30\% vs. 9\%, $\mathrm{P}=0.016)$; however, the SBRT group had a higher rate of prior lobar resection, which could predispose patients to clinical understaging if patients had metastatic tumors rather than the second primary tumors. In our study, there were no significant difference between matched groups in local, regional and distance control, supporting the notion that SBRT could be used as an alternative treatment to SLR.

The type and severity of post-treatment complications in potentially operable patients appeared to differ SBRT and SLR groups. In SBRT patients, adverse events were mostly mild and respiratory related, while SLR patient experienced more frequent and severe cardiovascular complications. SBRT-related complications were mild and similar to previously reported rates in similar populations: rates of grades 2 and $\geq 3$ radiation pneumonitis have been reported as $13.6 \%$ and $1.1 \%$ to $2 \%$ of the patients; rib fractures have been reported to occur in approximately $3 \%$ to $4.6 \%$ of the patients; and grade 3 dermatitis has been observed in 3.4\% of the patients. Adverse events might be more frequent and severe in medically inoperable patients with higher prescription dose in fewer fractions (54 Gy in 3 fractions) (16). Perioperative mortality and morbidity in SLR-treated NSCLC patients with high surgical risk were moderate and similar to a previous randomized clinical trial (Z4032) where nine (12\%) of patients reported grade 3 respiratory adverse events. Rates of perioperative mortality have been reported to be in $1.4 \%$ in some cases (26). In a study using the SEER-Medicare, unadjusted mortality of SLR at 30 and 90 days was $1.2 \%$ and $4.1 \%$, respectively (9). There was one treatment-related death after SLR in present work, and SBRT was found to decrease treatment-related toxicities compared with SLR.

In the present study, patients were well-matched at baseline. Meanwhile, variables that were not matchedincluding prior lobar resection and CCI-were also not significantly different. Furthermore, survival and disease control after SBRT in our study were consistent with results of previous studies, as SBRT achieved high local control rates ranging from $85 \%$ to $96 \%$ and 3 -year OS was $76 \%$ to $95 \%$ for patients with potentially operable early-stage NSCLC $(20,21,35)$. Our outcomes after SLR were similar to a phase III randomized trial (ACOSOG Z4032) for highrisk operable NSCLC (26). Our research is based on the Chinese population and has a larger research sample size.

Several limitations of the present study should be acknowledged. Firstly, treatment decision of NSCLC patients relied on accurate staging. SLR is preferred over SBRT to determine nodal status (6). We allowed for tumors up to $5 \mathrm{~cm}$ in size to be included in the study (T1-2a N0M0 NSCLC according to the 7th Edition of AJCC Staging Criteria), which increased the risk of nodal disease and recurrence rates and would lead to uncertainty. Potential patients in a disease stage more advanced than stage I, who were associated with poorer prognosis (36), were excluded after SLR, therefore outcomes might favor of SLR. As patient selection was conducted by routine examination of PET/CT and appropriate use of invasive staging of mediastinal and hilar nodes with mediastinoscopy 
or endobronchial ultrasound (EBUS) in high-risk subpopulations with nodal metastases, it achieved higher accuracy and specificity in nodal staging $(37,38)$. Secondly, as some radiation-induced changes arise from inflammation and fibrosis, timely evaluation of tumor recurrences after SBRT can be limited. Thirdly, the surgical techniques employed in this study were significantly heterogenous, making comparison and generalizability of results challenging. Fourthly, selection bias in treatment decisionmaking is unavoidable due to the different characteristics of each treatment modality and the nature of retrospective study; although PSM was applied to reduce differences in patients between SBRT and SLR groups at baseline, these differences could not be eliminated completely in a single institutional retrospective study. Number of variables used in PSM and number of patients was less than those analyses, which were based on population-based database. Thus, prospective randomized trials are still required.

\section{Conclusions}

These results suggest that SBRT can achieve similar disease control and survival to SLR in patients with clinical stage I NSCLC who are considered to be high-risk lobectomy patients. Both treatment strategies also appear to be well tolerated by patients, however treatment-related complications may be milder in SBRT. SBRT can be used as an alternative treatment option to SLR in high-risk operable patients. These findings should be confirmed in future prospective studies.

\section{Acknowledgments}

Funding: This work was supported by the Science Research Foundation of China Ministry of Health - Zhejiang Medicine \& Health Key Research Fund (No. 201339868).

\section{Footnote}

Reporting Checklist: The authors have completed the STROBE reporting checklist. Available at http://dx.doi. org/10.21037/jtd-21-339

Data Sharing Statement: Available at http://dx.doi. org/10.21037/jtd-21-339

Conflicts of Interest: All authors have completed the ICMJE uniform disclosure form (available at http://dx.doi. org/10.21037/jtd-21-339). The authors have no conflicts of interest to declare.

Ethical Statement: The authors are accountable for all aspects of the work in ensuring that questions related to the accuracy or integrity of any part of the work are appropriately investigated and resolved. The Ethics Review Committee of Zhejiang Cancer Hospital approved this study. The study is conformed to the provisions of the Declaration of Helsinki (as revised in 2013), and all participants were informed consent before taking part in the research.

Open Access Statement: This is an Open Access article distributed in accordance with the Creative Commons Attribution-NonCommercial-NoDerivs 4.0 International License (CC BY-NC-ND 4.0), which permits the noncommercial replication and distribution of the article with the strict proviso that no changes or edits are made and the original work is properly cited (including links to both the formal publication through the relevant DOI and the license). See: https://creativecommons.org/licenses/by-nc-nd/4.0/.

\section{References}

1. Sung H, Ferlay J, Siegel RL, et al. Global cancer statistics 2020: GLOBOCAN estimates of incidence and mortality worldwide for 36 cancers in 185 countries. CA Cancer J Clin 2021. [Epub ahead of print]. doi: 10.3322/caac.21660.

2. Bilano V, Gilmour S, Moffiet T, et al. Global trends and projections for tobacco use, 1990-2025: an analysis of smoking indicators from the WHO Comprehensive Information Systems for Tobacco Control. Lancet 2015;385:966-76.

3. National Lung Screening Trial Research Team, Aberle DR, Adams AM, et al. Reduced lung-cancer mortality with low-dose computed tomographic screening. N Engl J Med 2011;365:395-409.

4. Smith BD, Smith GL, Hurria A, et al. Future of cancer incidence in the United States: burdens upon an aging, changing nation. J Clin Oncol 2009;27:2758-65.

5. Ginsberg RJ, Rubinstein LV. Randomized trial of lobectomy versus limited resection for T1 N0 non-small cell lung cancer. Lung Cancer Study Group. Ann Thorac Surg 1995;60:615-22; discussion 622-3.

6. Shirvani SM, Jiang J, Chang JY, et al. Lobectomy, sublobar resection, and stereotactic ablative radiotherapy for earlystage non-small cell lung cancers in the elderly. JAMA 
Surg 2014;149:1244-53.

7. Rosen JE, Salazar MC, Wang Z, et al. Lobectomy versus stereotactic body radiotherapy in healthy patients with stage I lung cancer. J Thorac Cardiovasc Surg 2016;152:44-54.e9.

8. Cornwell LD, Echeverria AE, Samuelian J, et al. Videoassisted thoracoscopic lobectomy is associated with greater recurrence-free survival than stereotactic body radiotherapy for clinical stage I lung cancer. J Thorac Cardiovasc Surg 2018;155:395-402.

9. Shirvani SM, Jiang J, Chang JY, et al. Comparative effectiveness of 5 treatment strategies for early-stage nonsmall cell lung cancer in the elderly. Int $\mathrm{J}$ Radiat Oncol Biol Phys 2012;84:1060-70.

10. Harada H, Okada M, Sakamoto T, et al. Functional advantage after radical segmentectomy versus lobectomy for lung cancer. Ann Thorac Surg 2005;80:2041-5.

11. Altorki NK, Yip R, Hanaoka T, et al. Sublobar resection is equivalent to lobectomy for clinical stage $1 \mathrm{~A}$ lung cancer in solid nodules. J Thorac Cardiovasc Surg 2014;147:75462; discussion 762-4.

12. Fan J, Wang L, Jiang GN, et al. Sublobectomy versus lobectomy for stage I non-small-cell lung cancer, a meta-analysis of published studies. Ann Surg Oncol 2012;19:661-8.

13. Wolf AS, Richards WG, Jaklitsch MT, et al. Lobectomy versus sublobar resection for small $(2 \mathrm{~cm}$ or less) non-small cell lung cancers. Ann Thorac Surg 2011;92:1819-23; discussion 1824-5.

14. Yendamuri S, Sharma R, Demmy M, et al. Temporal trends in outcomes following sublobar and lobar resections for small $(</=2 \mathrm{~cm})$ non-small cell lung cancers--a Surveillance Epidemiology End Results database analysis. J Surg Res 2013;183:27-32.

15. Sun B, Brooks ED, Komaki RU, et al. 7-year follow-up after stereotactic ablative radiotherapy for patients with stage I non-small cell lung cancer: Results of a phase 2 clinical trial. Cancer 2017;123:3031-9.

16. Timmerman R, Paulus R, Galvin J, et al. Stereotactic body radiation therapy for inoperable early stage lung cancer. JAMA 2010;303:1070-6.

17. Murray L, Ramasamy S, Lilley J, et al. Stereotactic Ablative Radiotherapy (SABR) in Patients with Medically Inoperable Peripheral Early Stage Lung Cancer: Outcomes for the First UK SABR Cohort. Clin Oncol (R Coll Radiol) 2016;28:4-12.

18. Ball D, Mai T, Vinod S, et al. MA 13.07 A Randomized Trial of SABR vs Conventional Radiotherapy for
Inoperable Stage I Non-Small Cell Lung Cancer: TROG09.02 (CHISEL). J Thorac Oncol 2017;12:S1853.

19. Haque W, Verma V, Polamraju P, et al. Stereotactic body radiation therapy versus conventionally fractionated radiation therapy for early stage non-small cell lung cancer. Radiother Oncol 2018;129:264-9.

20. Nagata Y, Hiraoka M, Shibata T, et al. Prospective Trial of Stereotactic Body Radiation Therapy for Both Operable and Inoperable T1N0M0 Non-Small Cell Lung Cancer: Japan Clinical Oncology Group Study JCOG0403. Int J Radiat Oncol Biol Phys 2015;93:989-96.

21. Chang JY, Senan S, Paul MA, et al. Stereotactic ablative radiotherapy versus lobectomy for operable stage I nonsmall-cell lung cancer: a pooled analysis of two randomised trials. Lancet Oncol 2015;16:630-7.

22. Fernando HC, Timmerman R. American College of Surgeons Oncology Group Z4099/Radiation Therapy Oncology Group 1021: a randomized study of sublobar resection compared with stereotactic body radiotherapy for high-risk stage I non-small cell lung cancer. J Thorac Cardiovasc Surg 2012;144:S35-8.

23. Charlson ME, Pompei P, Ales KL, et al. A new method of classifying prognostic comorbidity in longitudinal studies: development and validation. J Chronic Dis 1987;40:373-83.

24. Schneider BJ, Daly ME, Kennedy EB, et al. Stereotactic Body Radiotherapy for Early-Stage Non-Small-Cell Lung Cancer: American Society of Clinical Oncology Endorsement of the American Society for Radiation Oncology Evidence-Based Guideline. J Clin Oncol 2018;36:710-9.

25. Grills IS, Mangona VS, Welsh R, et al. Outcomes after stereotactic lung radiotherapy or wedge resection for stage I non-small-cell lung cancer. J Clin Oncol 2010;28:928-35.

26. Fernando HC, Landreneau RJ, Mandrekar SJ, et al. The impact of adjuvant brachytherapy with sublobar resection on pulmonary function and dyspnea in high-risk patients with operable disease: preliminary results from the American College of Surgeons Oncology Group Z4032 trial. J Thorac Cardiovasc Surg 2011;142:554-62.

27. Port JL, Parashar B, Osakwe N, et al. A propensitymatched analysis of wedge resection and stereotactic body radiotherapy for early stage lung cancer. Ann Thorac Surg 2014;98:1152-9.

28. Matsuo Y, Chen F, Hamaji M, et al. Comparison of long-term survival outcomes between stereotactic body radiotherapy and sublobar resection for stage I non-smallcell lung cancer in patients at high risk for lobectomy: A propensity score matching analysis. Eur J Cancer 
2014;50:2932-8.

29. Ezer N, Veluswamy RR, Mhango G, et al. Outcomes after Stereotactic Body Radiotherapy versus Limited Resection in Older Patients with Early-Stage Lung Cancer. J Thorac Oncol 2015;10:1201-6.

30. Paul S, Lee PC, Mao J, et al. Long term survival with stereotactic ablative radiotherapy (SABR) versus thoracoscopic sublobar lung resection in elderly people: national population based study with propensity matched comparative analysis. BMJ 2016;354:13570.

31. Yerokun BA, Yang CFJ, Gulack BC, et al. A national analysis of wedge resection versus stereotactic body radiation therapy for stage IA non-small cell lung cancer. J Thorac Cardiovasc Surg 2017;154:675-686.e4.

32. Onishi H, Araki T, Shirato H, et al. Stereotactic hypofractionated high-dose irradiation for stage I nonsmall cell lung carcinoma: clinical outcomes in 245 subjects in a Japanese multiinstitutional study. Cancer 2004;101:1623-31.

33. Kestin L, Grills I, Guckenberger M, et al. Dose-response relationship with clinical outcome for lung stereotactic body radiotherapy (SBRT) delivered via online image guidance. Radiother Oncol 2014;110:499-504.

Cite this article as: Yuan XS, Chen WC, Lin QR, Liu YJ, Zhu YY, Sun XJ, Wu QY, Liu JS, Xu YP. A propensity-matched analysis of stereotactic body radiotherapy and sublobar resection for stage I non-small cell lung cancer in patients at high risk for lobectomy: the results in a Chinese population. J Thorac Dis 2021;13(3):1822-1832. doi: 10.21037/jtd-21-339
34. Shiue K, Cerra-Franco A, Shapiro R, et al. Histology, Tumor Volume, and Radiation Dose Predict Outcomes in NSCLC Patients After Stereotactic Ablative Radiotherapy. J Thorac Oncol 2018;13:1549-59.

35. Eriguchi T, Takeda A, Sanuki N, et al. Stereotactic body radiotherapy for operable early-stage non-small cell lung cancer. Lung Cancer 2017;109:62-7.

36. Rami-Porta R, Bolejack V, Crowley J, et al. The IASLC Lung Cancer Staging Project: Proposals for the Revisions of the T Descriptors in the Forthcoming Eighth Edition of the TNM Classification for Lung Cancer. J Thorac Oncol 2015;10:990-1003.

37. Gao SJ, Kim AW, Puchalski JT, et al. Indications for invasive mediastinal staging in patients with early nonsmall cell lung cancer staged with PET-CT. Lung Cancer 2017;109:36-41.

38. Um SW, Kim HK, Jung SH, et al. Endobronchial Ultrasound versus Mediastinoscopy for Mediastinal Nodal Staging of Non-Small-Cell Lung Cancer. J Thorac Oncol 2015;10:331-7.

(English Language Editor: M. Hawkins) 